\title{
Hemodynamic and Lactate Response to Exhaustive Exercise with L-Arginine Supplementation
}

Original Research

\begin{abstract}
Matthew Feeback ${ }^{1}$, Bailey Reitsma ${ }^{1}$
${ }^{1}$ Grand Valley State University, Allendale, MI/USA

Open Access

Abstract

Introduction: To further understand the effects of L-arginine on both its ability to enhance lactate clearance and increase overall blood flow before, during, and after exhaustive anaerobic exercise.

Methods: Twelve healthy male subjects between the ages of 18-25 first completed an initial visit where baseline data was recorded. Subjects completed three additional visits, in which they ingested either a placebo, two-gram or four-gram dose of Larginine. Blood flow $(\mathrm{BF})$ and lactate were recorded before ingestion of the treatment, 5 and 15-minutes post-ingestion prior to performing a push-up test to volitional fatigue. Immediately following the push-up test, $\mathrm{BF}$ and lactate were assessed and again 15-minutes post exercise.

Results: Blood lactate did not differ across condition $(\mathrm{p}=0.569)$. Lactate clearance was not influenced by L-arginine when analyzing the Area Under Curve. Blood flow increased with ingestion of the four-gram dose while at rest (the 15-minute mark). Blood flow was also enhanced in the four-gram dose immediately after exercise at the 25-minute mark.

Conclusions: The data suggests that a four-gram dose of L-arginine plays a more significant role in blood flow than the clearance of lactate after exercise compared to a two-gram dose or placebo.
\end{abstract}

Key Words: muscular endurance, blood flow, nitric oxide

Corresponding author: Matthew R. Feeback; feebacma@gvsu.edu

Nutrition: 2022, Volume

5 (Issue 1): 4

\section{Introduction}

Lactate accumulation during exercise is a key limiting factor during exhaustive exercise, this especially true in high-intensity activities. These high-intensity activities will especially rely heavily on anaerobic glycolysis, and subsequently yields lactate as a byproduct ${ }^{1}$. Exaggerated high-intensity exercise lends itself to the production of lactate due to the energy demands of the activity and an oxygen insufficiency. The increase in lactate will also coincides with increased hydrogen $(\mathrm{H}+)$ ion concentration. Lactate accumulation, along with the increase $\mathrm{H}+$ can disrupt the intracellular environment, resulting in reductions of force generation by the skeletal muscle and ensuing fatigue ${ }^{2,3}$.

L-arginine is a traditional non-essential amino acid that has consistently been recognized as a vasodilator and acts as a precursor for nitric oxide $(\mathrm{NO})^{4,5}$. Much research involving L-arginine has centered around its effects within clinical populations $\mathrm{s}^{4,67}$. However, research has emerged into $\mathrm{L}$-arginine as an ergogenic aid in healthy populations ${ }^{8}$. L-arginine is a vital component in the L-arginine-nitric oxide pathway ${ }^{4}$. This pathway describes the metabolism of L-arginine via 
nitric oxide synthase into NO. NO is a vasoactive substance that when released from the endothelium will illicit an inhibitory response upon the smooth muscle of the vascular system, the resultant is a vasodilatory response. Furthermore, the metabolism of L-arginine by way of nitric oxide synthase has also been demonstrated to yield 1citrulline?. At the cellular level within the cytoplasm, L-arginine can be converted into l-citrulline which may in turn play an important role in the diminishment of lactate by "buffering ammonia through the urea cycle, thereby enhancing the aerobic utilization of pyruvate"?.

It has been accepted that increased perfusion to skeletal muscle enhances lactate clearance through the increasing metabolism of the citric acid cycle ${ }^{3}$. As previously stated, lactate accumulation, along with $\mathrm{H}+$ increases have been linked to decreasing anaerobic power and subsequent fatigue. The proposition that the increasing blood flow response with exogenous L-arginine intake, subsequent $\mathrm{NO}$, and l-citrulline production will provide an ergogenic effect. Research in this area remains equivocal ${ }^{10}$. The augmented NO via L-arginine intake "before resistance training does not change the hemodynamic and vascular responses to resistance exercise" 11 . Other research also supports the augmented NO does not improve blood flow with exercise ${ }^{12,13}$. Still, it has also been reported that inhibition of NO did reduce blood flow to skeletal muscle during exercise ${ }^{14-16}$. It also has been observed that intravenous L-arginine administration reduced exercise induced lactate and ammonia concentrations ${ }^{17}$.

The purpose of this study was to examine the effect of exogenous L-arginine supplementation and its potential impact as an ergogenic aid by specifically examining muscular endurance, lactate concentration, and blood flow. Investigators hypothesized that with L-arginine supplementation, muscular endurance would be enhanced in conjunction with an increased blood flow and clearance of lactate.

\section{Scientific Methods}

Participants

Twelve male subjects aged 18-25 were recruited for participation in the study via direct contact with one of the members of the research team. All subjects read and signed an IRB-approved informed consent before their participation in the study. All methods and forms were approved by the Institutional Review Board at Grand Valley State University.

All subjects were non-smokers and free of any cardiovascular, respiratory, or metabolic disease. Additionally, the subjects were excluded if they had any previous experienced syncope or other relative contraindication of exercise.

Table 1. Subject Characteristics

\begin{tabular}{lc}
\hline Age (yrs) & $20.7 \pm 1.1$ \\
Height $(\mathrm{cm})$ & $178.6 \pm 7.9$ \\
Weight $(\mathrm{kg})$ & $76.8 \pm 9.7$ \\
Body Fat $\%$ & $11.2 \pm 3.1$ \\
Resting HR (bpm) & $67.1 \pm 11.9$ \\
Resting Mean Arterial Pressure & $90.8 \pm 4.6$ \\
VO2 max (ml.kg.min) & $47.3 \pm 7.1$ \\
\hline \multicolumn{2}{c}{$\mathrm{n}=12$. Data listed as mean \pm SD. }
\end{tabular}

Protocol

Subjects completed four total visits to the Human Performance Lab at Grand Valley State University.

Introduction and baseline measurements (Visit I)

These procedures included a completion of the informed consent and health history questionnaire. Resting measures were also recorded that included heart rate and blood pressure. In addition to the resting measures, anthropometric measurements were also taken that included height, weight, and body fat percentage, which was calculated via a 7-site skinfold measurement.

Lastly, all subjects completed a $\mathrm{VO}_{2}$ max test via a Parvo metabolic cart to volitional fatigue using a progressing cycle ergometer test. 
Experimental Procedure (Visits II, III and IV)

Treatments

The procedure for Visits II, III and IV were all identical apart from the treatment that was administered. The treatments included the administration of a 1) two-gram dose of L-arginine, 2) a four-gram dose of L-arginine or 3) a taste-matched placebo. All treatments were given to subjects in a manner that they would not be able to discern any variance across the treatments. Ensuring this, all treatments were mixed with eight ounces of water and a commercially available non-nutritive lemonade flavored drink mix. To limit bias, the investigator who administered the treatments was blinded as to which treatment was provided to subjects. All treatments were administered in a counterbalanced order using a Latin square to provide equal distribution across Visits II, III and IV.

\section{Measurements}

Participants reported to the Human Performance Lab at Grand Valley State in the mornings following a minimum of a 12 -hour fast and in the post-absorptive state. Upon entry to the lab and after a five-minute (min) rest period (-5$\mathrm{min}$ ), resting baseline measurements $(0-\mathrm{min})$ were recorded that included blood flow (blood velocity and vessel diameter) and blood lactate level. Following the collection of the resting measurements, the subjects ingested one of the three treatments ( 0 -min) as previously described. After ingestion of the treatment, the subjects remained in a resting and seated position for 15-minutes. Following this resting time, the same resting measurements were again taken and recorded (15-min). Following collection of the resting measures, subjects then completed a push-up test to exhaustion. At the conclusion of the push-up test, recovery measurements (lactate and blood flow) were collected and recorded (25-min). Subjects then remained in a resting state while in recovery for a duration of 10-min. After completing this 10-min recovery, the final series of recovery measurements were collected and recorded (40-min).

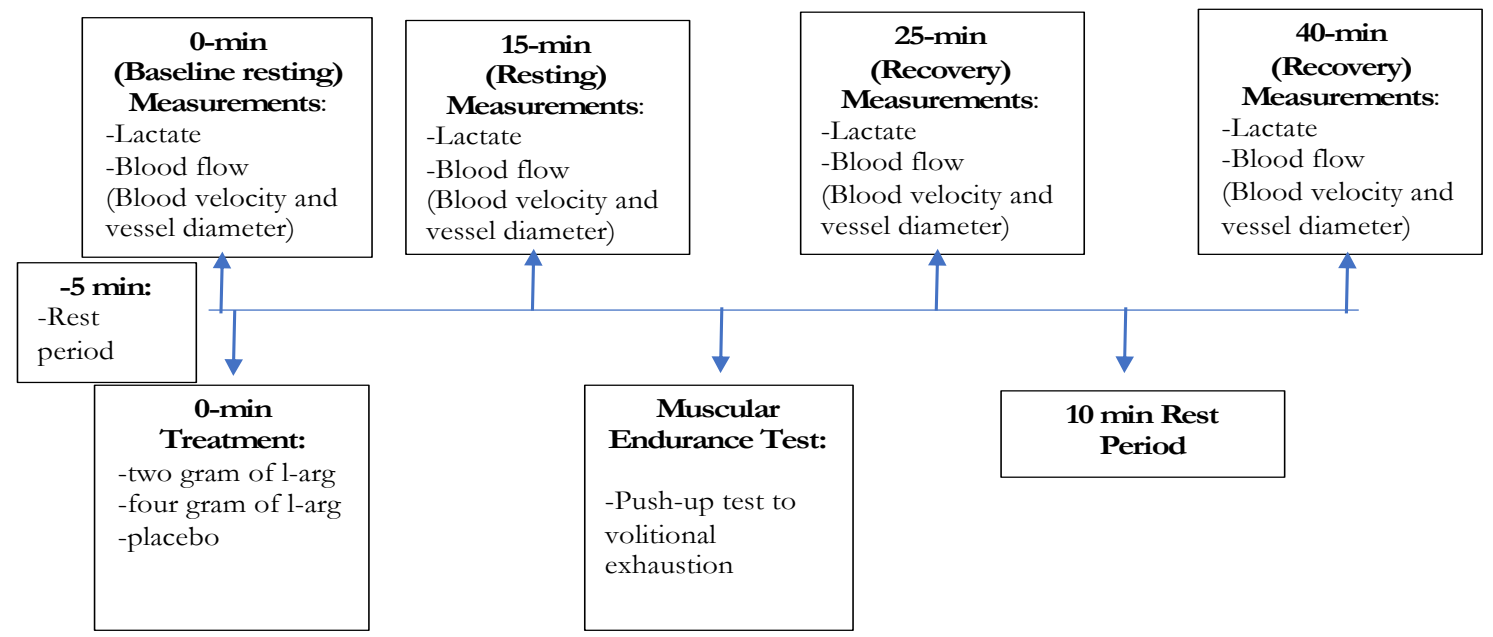

Figure 1. Schematic of timeline for experimental procedures.

Instrumentation and Description of Measurements Doppler Ultrasound

The doppler ultrasound procedures and calculations of blood flow was in accordance with guidelines that have been published ${ }^{17,18}$. Doppler ultrasound was utilized to assess blood velocity and vessel diameter of the brachial artery simultaneously using a linear array transducer operating in duplex mode with imaging frequency of $13 \mathrm{MHz}$ and Doppler frequency of $5 \mathrm{MHz}$ (Acuson X3000, Siemens Medical Solutions USA, Malvern, PA). The measurements were obtained with the probe in the appropriate position to maintain an isonation angle of $\leq 60^{\circ}$. The brachial artery was isonated at the approximate center region of the antecubital and axillary regions. A longitudinal image of the brachial artery was found to assess blood velocity $\left(\mathrm{V}_{\text {mean }}\right)$ and arterial diameter were attained while resting and for a duration of $1 \mathrm{~min}$. Vessel diameters were determined by averaging the perpendicular distance between the superficial and deep walls of the brachial artery at three locations along the brachial artery.

Blood flow was calculated via the standard equation of:

Blood flow $(\mathrm{ml} / \mathrm{min})=$ Velocity mean $\left(\mathrm{V}_{\text {mean }}\right) \times \pi \times$ vessel diameter $\left(\mathrm{V}_{\mathrm{d}} / 2\right]^{2} \times 60$

Lactate 
The lactate plus test meter (Nova Biomedical, Waltham MA) was used for analyses. Lactate was collected and analyzed using the highest of safety measures. A small finger prick was used to draw the blood needed for collection and analyses.

Muscular Endurance Test

The protocol for the push-up test was developed to incorporate the American College of Sports Medicine standards ${ }^{19}$. Push-ups were performed by using a standard starting "up" position, which includes the hands slightly wider than shoulder width with fingers pointing forward and in line with the level of the shoulder with the head, neck and back in line. The participant lowered the body by bending the elbows until his sternum was approximately 3-4 inches from the ground. Participants completed the push-ups at their respective self-pace, without a time limit and were allowed to rest in the "up" position. The self-pacing and no time limit were in accordance with LaChance \& Hortobayi that reported total repetitions completed and duration until fatigue ${ }^{20}$. Only pushups that incorporated correct technique were tallied in the total count. Total push-ups were recorded and timed until volitional fatigue or until the subject could not maintain proper technique on two consecutive repetitions.

\section{Statistical Analysis}

All statistical analyses were performed with IBM SPSS Statistics24 or Graphpad Prism Version 9. The use of three treatments (two grams of L-arginine, four grams of L-arginine and a control) was utilized. Additionally, four time points ( $0 \mathrm{~min}, 15 \mathrm{~min}, 25 \mathrm{~min}$, and $40 \mathrm{~min}$ ) were collected to be analyzed for the variables of blood flow (blood velocity and diameter of vessel) and blood lactate level. As such, a three-treatment $\mathrm{x}$ four time points repeated-measures ANOVA was utilized to determine any main effects of treatments and time points. Mauchly's test of sphericity was used to determine sphericity. When the sphericity assumption was violated, Greenhouse-Geisser corrections were applied, and was applied in the lactate and blood flow analyses. Effect sizes are expressed as Partial Eta Squared $\left(\eta^{2}\right)$, where $\eta^{2} \geq 0.01$ indicates a small effect, $\eta^{2} \geq 0.06$ indicates a medium effect and $\eta^{2} \geq 0.14$ indicates a large effect. Pairedsamples T-test were utilized to assess any difference at specific time points. A one-way ANOVA applied in the analysis of the muscular endurance test (push-ups to volitional fatigue). Statistical significance was set at a 95\% significant level $(\mathrm{p}<0.05)$.

\section{Results}

\section{Lactate}

A Repeated Measures ANOVA with a Greenhouse-Geisser correction determined that the main effect for time differed for mean lactate concentrations $F(2.056,67.852)=93.615, p<0.001, \eta^{2}=0.050$, but did not for conditionby-time interaction $(\mathrm{p}=.569)$. Area under curve (AUC) analysis was also conducted to better illustrate clearance kinetics (see Table 2A). No significant differences where observed with the AUC (see Table 2B).

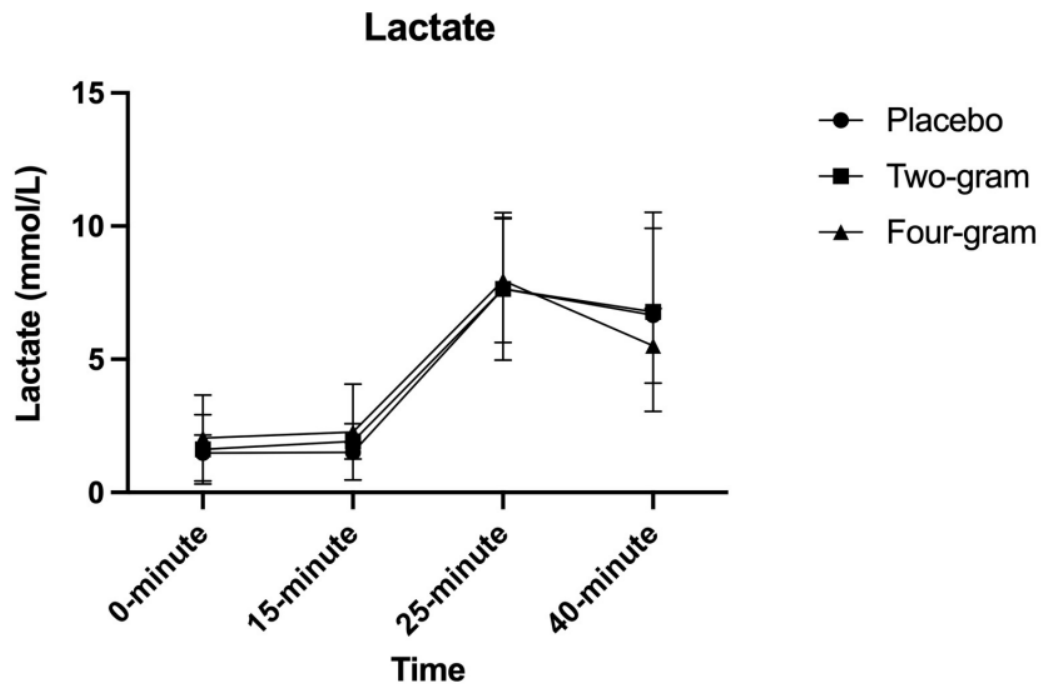

Figure 2. Blood lactate concentration across time. 
Table 2A. Area under curve of Lactate $(\mathrm{mmol} / \mathrm{L})$.

\begin{tabular}{ll}
\hline Treatment & \\
Placebo & $13.2 \pm 2.6$ \\
Two-gram & $13.7 \pm 2.7$ \\
Four-gram & $14.0 \pm 2.3$ \\
\hline
\end{tabular}

Data listed as mean \pm SD.

Table 2B. Statistical P-values Between Area Under Curve.

\begin{tabular}{ll}
\hline Comparisons & P-values \\
Placebo/Two-gram & .366 \\
Placebo/Four-gram & .227 \\
Two-gram/Four-gram & .182 \\
\hline
\end{tabular}

Blood Flow

A Repeated Measures ANOVA with a Greenhouse-Geisser correction determined that the main effect for time differed for mean blood flow $\left(\mathrm{F}(1.324,43.7)=32.217 \mathrm{p}<0.001, \eta^{2}=.066\right)$.

Paired samples T-tests identified a significant difference at the 15-min mark (post-treatment, while resting) between the four-gram dose as compared to both the placebo $(69.05 \pm 17.46 \mathrm{vs} 49.46 \pm 18.05 \mathrm{ml} / \mathrm{min}$, respectively, where a $\mathrm{p}-$ value $=.022)$ and two-gram treatment $(69.05 \pm 17.46 \mathrm{vs} 53.87 \pm 20.57 \mathrm{ml} / \mathrm{min}$, respectively, where $\mathrm{p}=.013)$.

Additionally, significance was also determined at the 25 -min mark (25-min post-treatment and immediately after exercise) between the two-gram and four-gram treatments $(96.20 \pm 40.44$ vs $133.35 \pm 47.21 \mathrm{ml} / \mathrm{min}$, respectively) where $\mathrm{p}=0.006$. No other significance was determined, see Figure 3.

\section{Blood Flow}

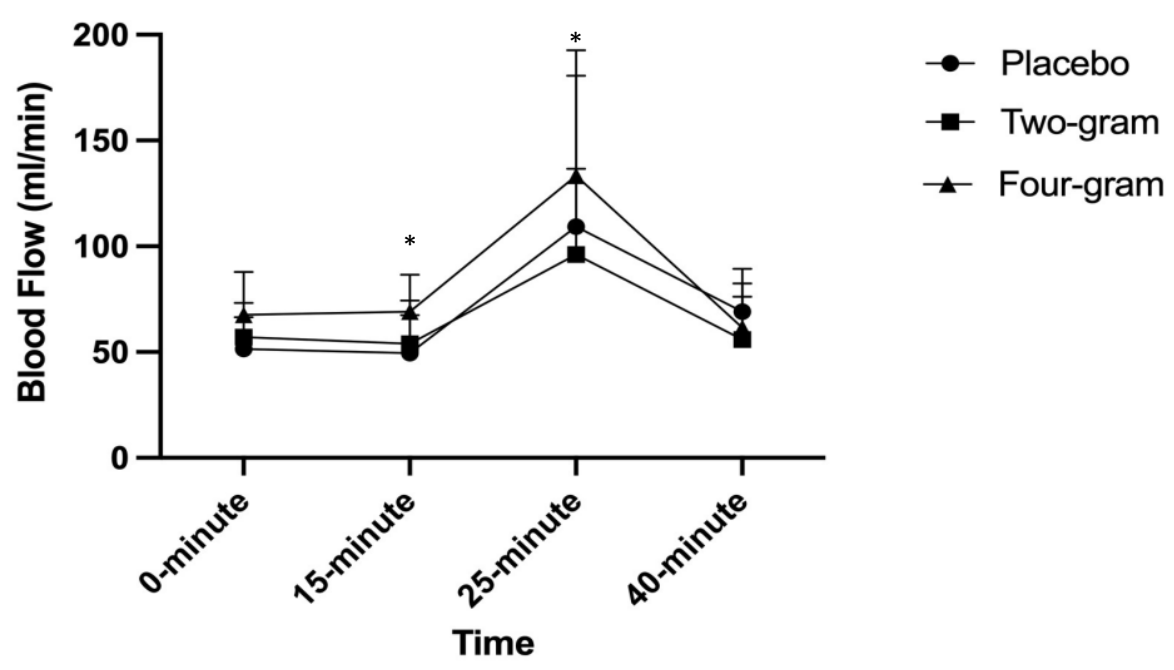

Figure 3. Blood flow across time and condition. Significance indicated by “*," where $p=0.022$ between four-gram and placebo \& $\mathrm{p}=.013$ between four-gram and two-gram treatments at the 15-min mark. Significance was also determined between four-gram and two-gram where $\mathrm{p}=0.006$ at the 25 -min mark.

Muscular Endurance Test

Table 3 provides representation of treatments for the a) push-up test to volitional fatigue, b) time to exhaustion and c) push-ups per second. No statistical significance was revealed when a One-way ANOVA was performed, in which pvalues yielded were push-ups $=0.969$; exhaustion time 0.884 ; and push-ups per second 0.600 . 
Table 3. Comparison of push-ups, exhaustion time and push-ups per second.

\begin{tabular}{lccc} 
Treatment & Push-ups & Exhaustion Time (seconds) & Push-ups/sec \\
a. Placebo & $61.7 \pm 8.9$ & $87.5 \pm 14.6$ & $.713 \pm .12$ \\
b. Two-gram & $61.0 \pm 6.2$ & $80.1 \pm 14.7$ & $.793 \pm .21$ \\
c. Four-gram & $58.8+9.0$ & $82.9+16.5$ & $.748+.21$ \\
\hline
\end{tabular}

Data listed as mean + SD.

\section{Discussion}

Results of this study indicate that L-arginine may play a significant role in the increase of blood flow while at rest and immediately after exhaustive exercise. This increase in blood flow did not seem to offer any enhanced lactate clearance that has previously been reported, most notably by Schaefer et al. that indicated that L-arginine reduced exerciseinduced plasma lactate ${ }^{21}$. The reduction in exercise-induced lactate discussed by Schaefer et al. could be in part due to the delivery method of L-arginine. Their delivery of L-arginine was done via intravenously, rather than orally. Intravenous delivery could provide a greater bioavailability as compared to oral administration, although the pharmokinetics of L-arginine is largely not understood ${ }^{22}$.

It has also been reported that an increased L-arginine load also increased 1-citrulline and nitric oxide, producing a vasodilatory effect. Nitric oxide has also been demonstrated to enhance guanylate cyclase and cGMP in the vascular smooth muscle cells, resulting in the inability to contract ${ }^{23}$. Increasing vasodilation augments blood flow due to the decreased vascular pressure. Hyperaemia through skeletal muscle also enhances the removal of lactate via an increased Cori Cycle activity ${ }^{24}$. Although, blood flow was augmented, data from the current investigation does not support an increased lactate clearance.

Again, blood flow data from the current investigation indicates an increased blood flow after ingestion of the fourgram dose of L-arginine as compared to the placebo and two-gram dose. There is supporting evidence that the hyperemic response being reported is dependent on endothelial derived nitric oxide (EDNO). This is evidenced in both human and animal models, as exercise hyperemia is curtailed when EDNO is minimized ${ }^{25,26}$. Although it is wellsupported that $\mathrm{L}$-arginine plays a vital role in the production of nitric oxide and in vasodilation, other research provides uncertainty to the efficacy of this mechanism within healthy populations.

As such, there is opposing research that suggests that L-arginine may have no significant effect on vasodilation in people, void of any vascular disorders ${ }^{27-29}$. Peripheral artery stiffness and blood flow increases after resistance exercise, but a seven-gram dose of L-arginine in conjunction with resistance exercise did not provide a substantial increased hemodynamics ${ }^{11}$. As outlined above, there is conflicting data as to the efficacy of L-arginine in enhancing hemodynamics after exercise, especially in a healthy population.

Again, L-arginine has recently been touted as an ergogenic aid that will improve muscular performance. Despite these claims, there is little scientific evidence that supports exogenous L-arginine will increase muscular performance. Campbell et al. states that males who consumed 12 grams of L-arginine/day for eight weeks did increase their 1-RM bench press and anaerobic power in the Wingate test ${ }^{30}$. However, much of the literature suggest that no ergogenic effect is found with L-arginine supplementation ${ }^{28}$.

Information from this investigation is reinforced with much of the work in L-arginine and muscular performance. The outcome from this study is there was not a positive, differential response when examining total push-ups or time to volitional exhaustion with consumption of L-arginine. Most similarly, Alvares et al. reported that Larginine increased blood volume at the level of the muscle but failed to enhance muscular strength or endurance after ingestion of a sixgram dose of L-arginine ${ }^{31}$. The lack of efficacy is not supportive of an ergogenic effect after exogenous L-arginine ingestion ${ }^{32-34}$. As these suggest, muscular power, muscular endurance, or recovery are not increased with L-arginine supplementation.

Future Research

It could be advantageous to use a dose of $\mathrm{L}$-arginine that is relative to body mass or lean body mass, especially since body weight is often going to predicate a dose of drugs administered clinically ${ }^{35}$. Other considerations would include but may not be limited to researching a chronic dosing, rather than the acute dose used in this study; the inclusion of a muscular endurance or power post-test to assess if L-arginine influences recovery because of lactate clearance. 
Furthermore, investigating l-citrulline or nitric oxide synthase would also provide information that could better explain the results and lead to more concrete information.

Limitations

Limitations within this project would lend itself to additional research. These limitations include a relatively small sample size; a larger sample size would yield a more vigorous investigation into the effects of L-arginine supplementation. The muscular endurance test, while standardized, did allow for self-pacing, which resulted in momentary resting in between repetitions for some subjects, while others did not take any rest periods.

\section{Media-Friendly Summary}

$\mathrm{L}$-arginine is publicized as an ergogenic aid to improve lactate clearance, blood flow dynamics, and improve muscular endurance and/or power. Results from this project are suggestive that a four-gram dose of L-arginine will enhance blood flow. Muscular endurance and lactate clearance were not improved with consumption of L-arginine.

\section{Acknowledgements}

The authors would like to thank each subject who participated in the study. Enormous gratitude is also owed to the numerous research assistants who helped with data collection. Authors would also like to thank the P. Douglas Kindschi Undergraduate Research Fellowship in the Sciences for the support in funding this research project.

Authors report no conflicts of interest.

\section{References}

1. Powers SK, Howley ET, Exercise Physiology: The Theory and Application to Fitness and Performance 10th ed. McGraw Hill; 2018.

2. Bangsbo J, Madsen K, Kiens B, Richter EA. Effect of muscle acidity on muscle metabolism and fatigue during intense exercise in man. J Physiol. 1996 Sep 1;495 ( Pt 2)(Pt 2):587-96. doi:10.1113/jphysiol.1996.sp021618.

3. McArdle WD, Katch FI, Katch VL, Exercise Physiology: Nutrition, Energy and Human Performance 8th ed. Wolters Kluwer; 2015.

4. Doutreleau S, Mettauer B, Piquard F, Schaefer A, Lonsdorfer E, Richard R, Geny B. Chronic but not acute oral Larginine supplementation delays the ventilatory threshold during exercise in heart failure patients. Can J Appl Physiol. 2005 Aug;30(4):419-32. doi: 10.1139/h05-131.

5. Böger RH. The pharmacodynamics of L-arginine. Altern Ther Health Med. 2014 May-Jun;20(3):48-54.

6. Mehta S, Stewart DJ, Langleben D, Levy RD. Short-term pulmonary vasodilation with L-arginine in pulmonary hypertension. Circulation. 1995 Sep 15;92(6):1539-45. doi: 10.1161/01.cir.92.6.1539.

7. Clarkson P, Adams MR, Powe AJ, Donald AE, McCredie R, Robinson J, McCarthy SN, Keech A, Celermajer DS, Deanfield JE. Oral L-arginine improves endothelium-dependent dilation in hypercholesterolemic young adults. J Clin Invest. 1996 Apr 15;97(8):1989-94. doi: 10.1172/JCI118632

8. Viribay A, Burgos J, Fernández-Landa J, Seco-Calvo J, Mielgo-Ayuso J. Effects of Arginine Supplementation on Athletic Performance Based on Energy Metabolism: A Systematic Review and Meta-Analysis. Nutrients. 2020 May 2;12(5):1300. doi: 10.3390/nu12051300.

9. Aguayo E, Martínez-Sánchez A, Fernández-Lobato B, Alacid F. L-Citrulline: A Non-Essential Amino Acid with Important Roles in Human Health. Applied Sciences. 2021; 11(7):3293. https://doi.org/10.3390/app11073293.

10. Gonzalez AM, Trexler ET Citrulline Supplementation on Exercise Performance in Humans: A Review of the Current Literature. J Strength Cond Res. 2020 May;34(5):1480-1495. doi: 10.1519/JSC.0000000000003426.

11. Fahs CA, Heffernan KS, Fernhall B. Hemodynamic and vascular response to resistance exercise with L-arginine. Med Sci Sports Exerc. 2009 Apr;41(4):773-9. doi: 10.1249/MSS.0b013e3181909d9d.

12. Bradley SJ, Kingwell BA, McConell GK. Nitric oxide synthase inhibition reduces leg glucose uptake but not blood flow during dynamic exercise in humans. Diabetes. 1999 Sep;48(9):1815-21. doi: 10.2337/diabetes.48.9.1815.

13. Hickner RC, Fisher JS, Ehsani AA, Kohrt WM. Role of nitric oxide in skeletal muscle blood flow at rest and during dynamic exercise in humans. Am J Physiol. 1997 Jul;273(1 Pt 2):H405-10.

doi: 10.1152/ajpheart.1997.273.1.H405.

14. Boushel R, Langberg H, Gemmer C, Olesen J, Crameri R, Scheede C, Sander M, Kjaer M. Combined inhibition of nitric oxide and prostaglandins reduces human skeletal muscle blood flow during exercise. J Physiol. 2002 Sep 1;543(Pt 2):691-8. doi: 10.1113/jphysiol.2002.021477. 
15. Mortensen SP, González-Alonso J, Damsgaard R, Saltin B, Hellsten Y. Inhibition of nitric oxide and prostaglandins, but not endothelial-derived hyperpolarizing factors, reduces blood flow and aerobic energy turnover in the exercising human leg. J Physiol. 2007 Jun 1;581(Pt 2):853-61. doi: 10.1113/jphysiol.2006.127423.

16. Schrage WG, Dietz NM, Joyner MJ. Effects of combined inhibition of ATP-sensitive potassium channels, nitric oxide, and prostaglandins on hyperemia during moderate exercise. J Appl Physiol (1985). 2006 May;100(5):1506-12. doi: 10.1152/japplphysiol.01639.2005.

17. Harris RA, Nishiyama SK, Wray DW, Richardson RS. Ultrasound assessment of flow-mediated dilation. Hypertension. 2010 May;55(5):1075-85. doi: 10.1161/HYPERTENSIONAHA.110.150821.

18. Wray DW, Witman MA, Ives SJ, McDaniel J, Trinity JD, Conklin JD, Supiano MA, Richardson RS.Hypertension. 2013 Aug;62(2):345-51. doi: 10.1161/HYPERTENSIONAHA.113.01578. Epub 2013 Jun 17.

19. American College of Sports Medicine. ACSM'S Health-Related Physical Fitness Assessment Manual. 4th ed. Philadelphia, PA: Wolters Kluwer/Lippincott Williams \& Wilkins; 2013. pp. 76-91.

20. LaChance PF, Hortobagyi T. Influence of cadence on muscular performance during push-up and pull-up exercises. J Strength Cond Res 1994; 8: 76-9.

21. Schaefer A, Piquard F, Geny B, Doutreleau S, Lampert E, Mettauer B, Lonsdorfer J. L-arginine reduces exerciseinduced increase in plasma lactate and ammonia. Int J Sports Med. 2002 Aug;23(6):403-7.

doi: 10.1055/s-2002-33743. PMID: 12215958.

22. Tangphao O, Grossmann M, Chalon S, Hoffman BB, Blaschke TF. Pharmacokinetics of intravenous and oral Larginine in normal volunteers. Br J Clin Pharmacol. 1999 Mar;47(3):261-6. doi: 10.1046/j.1365-2125.1999.00883.x.

23. Yaman, H., Tiryaki-Sönmez, G. , \& Gürel, K. (2010). Effects of oral L-arginine supplementation on vasodilation and VO2 max in male soccer players. Biomedical Human Kinetics, 2(1), 25-29. doi:10.2478/v10101-010-0006-x

24. Brooks GA. The lactate shuttle during exercise and recovery. Med Sci Sports Exerc. 1986 Jun;18(3):360-8. doi: 10.1249/00005768-198606000-00019.

25. Dyke CK, Proctor DN, Dietz NM, Joyner MJ. Role of nitric oxide in exercise hyperaemia during prolonged rhythmic handgripping in humans. J Physiol. 1995 Oct 1;488 (Pt 1)(Pt 1):259-65.

doi: 10.1113/jphysiol.1995.sp020964

26. Hirai T, Visneski MD, Kearns KJ, Zelis R, Musch TI. Effects of NO synthase inhibition on the muscular blood flow response to treadmill exercise in rats. J Appl Physiol (1985). 1994 Sep;77(3):1288-93.

doi: $10.1152 /$ jappl.1994.77.3.1288.

27. Chin-Dusting JP, Alexander CT, Arnold PJ, Hodgson WC, Lux AS, Jennings GL. Effects of in vivo and in vitro L-arginine supplementation on healthy human vessels. J Cardiovasc Pharmacol. 1996 Jul;28(1):158-66. doi: 10.1097/00005344-199607000-00023.

28. Liu TH, Wu CL, Chiang CW, Lo YW, Tseng HF, Chang CK. No effect of short-term arginine supplementation on nitric oxide production, metabolism and performance in intermittent exercise in athletes. J Nutr Biochem. 2009 Jun;20(6):462-8. doi: 10.1016/j.jnutbio.2008.05.005.

29. Adams MR, Forsyth CJ, Jessup W, Robinson J, Celermajer DS. Oral L-arginine inhibits platelet aggregation but does not enhance endothelium-dependent dilation in healthy young men. J Am Coll Cardiol. 1995 Oct;26(4):1054-61. doi: 10.1016/0735-1097(95)00257-9.

30. Campbell B, Roberts M, Kerksick C, Wilborn C, Marcello B, Taylor L, Nassar E, Leutholtz B, Bowden R, Rasmussen C, Greenwood M, Kreider R. Pharmacokinetics, safety, and effects on exercise performance of L-arginine alpha-ketoglutarate in trained adult men. Nutrition. 2006 Sep;22(9):872-81.

doi: $10.1016 /$ j.nut.2006.06.003

31. Alvares TS, Conte CA, Paschoalin VM, Silva JT, Meirelles Cde M, Bhambhani YN, Gomes PS. Acute 1-arginine supplementation increases muscle blood volume but not strength performance. Appl Physiol Nutr Metab. 2012 Feb;37(1):115-26.

doi: $10.1139 /$ h11-144.

32. Andrade WB, Jacinto JL, da Silva DK, Roveratti MC, Estoche JM, Oliveira DB, Balvedi MCW, da Silva RA, Aguiar AF. 1-Arginine supplementation does not improve muscle function during recovery from resistance exercise. Appl Physiol Nutr Metab. 2018 Sep;43(9):928-936. doi: 10.1139/apnm-2017-0594.

33. Wax B, Mayo JJ, Hilton LA, Mareio HC, Miller JD, Webb HE, Lyons B (2013) Acute ingestion of L-arginine alphaketoglutarate fails to improve muscular strength and endurance in ROTC cadets. Int J Exerc Sci 6:91-97

34. Wax B, Kavazis AN, Webb HE, Brown SP. Acute L-arginine alpha ketoglutarate supplementation fails to improve muscular performance in resistance trained and untrained men. J Int Soc Sports Nutr. 2012 Apr 17;9(1):17. doi: 10.1186/1550-2783-9-17.

35. Sheiner LB, Ludden TM. Population pharmacokinetics/dynamics. Annu Rev Pharmacol Toxicol. 1992;32:185-209. doi: 10.1146/annurev.pa.32.040192.001153. 
